\title{
Rational Study Endpoint(s) for Preoperative Trials in Pancreatic Cancer: Pathologic Response Rate, Margin Negative Resection, Overall Survival or 'All of the Above'?
}

\author{
Gauri R. Varadhachary, MBBS, $\mathrm{MD}^{1}$ and Douglas B. Evans, $\mathrm{MD}^{2}$ \\ ${ }^{1}$ Division of Cancer Medicine, The University of Texas M. D. Anderson Cancer Center, Houston, TX; ${ }^{2}$ Department of \\ Surgery, The Medical College of Wisconsin, Milwaukee, WI
}

In this issue of Annals of Surgical Oncology, Van Buren and colleagues present the results of their phase II trial of preoperative fixed-dose rate gemcitabine combined with bevacizumab and accelerated 30 Gy radiotherapy (RT) for patients with potentially resectable (or borderline resectable) pancreatic ductal adenocarcinoma (PDC). ${ }^{1}$ Of the 58 evaluable patients, 4 (7\%) demonstrated metastatic progression on post-treatment/preoperative restaging $\mathrm{CT}$ and another $11(20 \%)$ were unresectable at the time of surgery due to distant metastases (10) or local tumor progression (1). A successful pancreatic resection was accomplished in $43(74 \%)$ of the 58 patients, and $19(44 \%)$ required vascular resection at the time of pancreatectomy. The R0 resection rate was $88 \%$. However, in $60 \%$ of the surgical specimens, the histopathologic assessment of treatment response was minimal; there was only 1 complete pathologic response to induction therapy. Median overall survival (OS) for the entire cohort was 16.8 months (95\% CI, 14.9-21.3 months) and 19.7 months (95\% CI, 16.5-28.2 months) for the 43 patients who completed all intended therapy to include surgery. The authors concluded that in spite of the high $\mathrm{R} 0$ resection rate, the low rate of complete pathologic response and the modest OS did not warrant further investigation of this regimen in larger phase II/III trials. Although viewed by many as a "negative" trial, the authors should be commended for completing a prospective study with such novel treatment sequencing, reporting their data on various

(C) Society of Surgical Oncology 2013

First Received: 26 April 2013;

Published Online: 14 August 2013

D. B. Evans, MD

e-mail: devans@mcw.edu aspects of the study including OS, and their thoughtful Discussion.

This study illustrates some important considerations in eligibility and study endpoints for preoperative trials involving patients with localized PDC. Given the sound biologic and clinical rationale for the preoperative approach to operable PDC, it is not surprising to see an evolving acceptance of this method. In the current era of FOLFIRINOX, nab-paclitaxel, and emerging immunotherapeutic agents, preoperative trials are currently in place or being planned for patients with localized PDC. Therefore, we urgently need to reach consensus on eligibility and valid study endpoints. To date, resection rate (completion of all intended therapy to include resection of the pancreatic tumor), pathologic response rate as assessed histologically in the tumor specimen, margin status, disease-free survival, and OS are the most commonly measured endpoints.

In the era of the multidetector CT optimized for pancreatic imaging, tumors of "borderline resectability" have emerged as a distinct subset of PDC. ${ }^{2}$ The attempt to standardize the definition of borderline resectable is a work in progress and has been modified with time through the National Comprehensive Cancer Network (NCCN), initial descriptions from M. D. Anderson Cancer Center, and consensus conferences, the first being sponsored by the AHPBA/SSAT/SSO. ${ }^{2-4}$ This distinction (between resectable and borderline resectable) is essential to minimize potentially confounding results of clinical trials. The study discussed herein allowed enrollment of patients with both resectable and borderline resectable disease; the later defined by some element of noncircumferential "involvement" of the portal vein. Approximately $50 \%$ of patients met borderline criteria as reflected in the $44 \%$ of patients who required portal vein resection at the time of surgery. 
Even though retrospective data from several single-institution reports suggest that vascular resection does not negatively affect survival, this remains controversial largely because vascular resection can occur as either a planned event (by an experienced operative team) or as the result of an inadvertent intraoperative misadventure; the latter situation often results in the need for rapid removal of the surgical specimen and a positive margin of resection., As clinical trial enrollment expands to include more than just the very highest volume institutions, entry criteria become even more important as the inclusion of patients with borderline tumors frequently results in the enrollment of patients with more advanced disease than can be managed surgically. If surgery is attempted, the complexity of the operation may be beyond the level of experience of the surgical team, thereby compromising the oncologic outcome of the procedure. ${ }^{7}$ When designing future prospective trials of novel preoperative approaches, standardization of the stage of disease for the study population is essential to allow for accurate interpretation of study results, specifically, resection rate, R0/1 margin rate, frequency of local recurrence, and OS.

Resection rate (the percent of patients that complete all intended neoadjuvant therapy, restaging, and surgical resection of the primary tumor) is an obvious endpoint as one would assume that the majority of patients have radiographically occult distant metastases at the time of diagnosis. If such metastases are sensitive to the induction therapy used, they should remain occult after neoadjuvant therapy, be they viable or nonviable (complete response), and there should be no evidence of disease progression at the time of post-treatment/preoperative restaging and at the time of planned pancreatectomy. Indeed, it is the ability to potentially sterilize distant disease when small and clinically unapparent that represents the "holy grail" of neoadjuvant treatment sequencing; this is more likely to occur in the setting of an immune competent host who has not experienced the stress/ trauma of a large operation. To the extent that distant disease has a response profile similar to regional lymph node metastases, a patient with micrometastatic disease may become "curable" based solely on treatment sequencing as the incidence of regional lymph node metastases declines considerably after induction therapy (70-80 \% with surgery first and 20-40\% after induction therapy). The margin of resection, otherwise termed the "margin negative" resection rate also has a place as an objective outcome metric for preoperative trials. The unique ability of PDC to spread along the perineural sheath that surrounds visceral arteries and the autonomic ganglia at the origins of the superior mesenteric and celiac arteries predisposes patients to local recurrence even when all gross tumor has been removed. The low rate of local recurrence reported in this and other trials is likely the result, in part, of preoperative chemoradiation, especially in patients with borderline disease. If one excludes $\mathrm{R} 2$ resections, the OS benefit of an R0 vs a (true) $\mathrm{R} 1$ resection remains to be determined. Although, one could argue that even an R0 resection does not imply a favorable long-term survival for most patients with pancreatic cancer, to the extent that it may reduce local-regional recurrence, it may have a positive impact on quality of life and disease-free survival. The margin negative resection rate and accurate analysis of subsequent sites of recurrence remain valuable metrics for preoperative trials especially as we try to obtain a better understanding of how best to sequence therapies and the role of RT.

If we believe that resectable PC is analogous to resectable rectal cancer, then complete or near-complete pathologic response in the surgical specimen is also applicable as an important outcome metric. However, probably because of the stromal barrier, complex intratumoral heterogeneity, and the PDC microenvironment, pathologic complete responses after surgical resection are rare and consistent with the minor (RECIST) tumor responses noted on post-treatment/preoperative imaging. In the Van Buren study, expecting a $10 \%$ path CR ( $\sim 6$ patients) seems like a challenging target especially without any comprehensive preclinical data to suggest this effect is achievable. Also, in our own preoperative studies of gemcitabine-based chemoradiation, a pathologic CR was achieved in only 1 of 116 patients; moreover, there were no statistically significant differences in overall survival by treatment effect scores. ${ }^{8,9}$

OS remains an independent important study endpoint for all pancreatic cancer trials. As we develop more effective systemic therapies for PDC, proceeding with surgery after a period of induction therapy will be even more compelling especially if there is a clear positive impact on OS. Given the morbidity of early recurrence (bowel obstruction, ascites, pain etc.), it is also important to note that a significant improvement in DFS (even without an impact on OS) may be an appropriate endpoint and should not be disregarded.

Ultimately, to make progress, all preoperative clinical trial designs for PDC should have standardized valid study endpoints, agreed on by consensus, which then allows meaningful comparisons between studies. In that regard, we look forward to the results of the intergroup Alliance (A0211101) trial, a pilot multi-institutional preoperative study in borderline patients, which will hopefully establish a research infrastructure upon which future trials can be based.

\section{REFERENCES}

1. Van Buren G II, Ramanathan RK, Krasinskas AM, Smith RP, Abood GJ, Bahary N, et al. Phase II study of induction fixed-dose rate gemcitabine and bevacizumab followed by 30 gy radiotherapy as 
preoperative treatment for potentially resectable pancreatic adenocarcinoma. Ann Surg Oncol. doi:10.1245/s10434-013-3161-9.

2. Appel BL, Tolat P, Evans DB, Tsai S. Current staging systems for pancreatic cancer. Cancer J. 2012;18:539-49.

3. Evans DB, Farnell MB, Lillemoe KD, Vollmer C Jr, Strasberg SM, Schulick RD. Surgical treatment of resectable and borderline resectable pancreas cancer: expert consensus statement. Ann Surg Oncol. 2009;16:1736-44.

4. Varadhachary GR, Tamm EP, Crane C, Evans DB, Wolff RA. Borderline resectable pancreatic cancer. Curr Treat Options Gastroenterol. 2005;8:377-84.

5. Christians K, Evans DB. Pancreaticoduodenectomy and vascular resection: persistent controversy and current recommendations. Ann Surg Oncol. 2009;16:789-91.

6. Tseng JF, Raut CP, Lee JE, Pisters PWT, Vauthey JN, Abdalla EK, et al. Pancreaticoduodenectomy with vascular resection: margin status and survival duration. J Gastrointest Surg. 2004;8:935-50.
7. Katz MHG, Fleming JB, Pisters PWT, Lee JE, Evans DB. Anatomy of the superior mesenteric vein with special reference to the surgical management of first-order branch involvement at pancreaticoduodenectomy. Ann Surg. 2008;248:1098-102.

8. Evans DB, Varadhachary GR, Crane CH, Sun CC, Lee JE, Pisters PWT, et al. Preoperative gemcitabine-based chemoradiation for patients with resectable adenocarcinoma of the pancreatic head. $J$ Clin Oncol. 2008;26:3496-502.

9. Varadhachary GR, Wolff RA, Crane CH, Sun CC, Lee JE, Pisters PWT, et al. Preoperative gemcitabine and cisplatin followed by gemcitabine-based chemoradiation for resectable adenocarcinoma of the pancreatic head. J Clin Oncol. 2008;26:3487-95. 